\title{
Coordination structure dominated performance of single-atomic Pt catalyst for anti-Markovnikov hydroboration of alkenes
}

\author{
$\mathrm{Qi} \mathrm{Xu}^{1 \dagger}$, ChenXi Guo ${ }^{2 \dagger}$, Shubo Tian ${ }^{1 \dagger}$, Jian Zhang ${ }^{1}$, Wenxing Chen ${ }^{3}$, Weng-Chon Cheong ${ }^{1}$, Lin Gu ${ }^{4}$, \\ Lirong Zheng ${ }^{5}$, Jianping Xiao ${ }^{2}$, Qiang Liu ${ }^{1}$, Bijie $\mathrm{Li}^{1}$, Dingsheng Wang ${ }^{1 *}$ and Yadong $\mathrm{Li}^{1}$
}

\begin{abstract}
The rational design of efficient single-atomic (SA) catalysts is essential and highly desirable but impeded by the lack of sufficient acknowledge between structure and property. To this end, it is critical to clarify the effect of the coordination structure of active metal centers on the catalytic activities for the design of such catalysts. Here, we report that different coordination structures of SA Pt catalysts can dramatically influence their activities for anti-Markovnikov hydroboration of alkenes. Compared with the other two coordination structures $\left(\mathrm{Pt}-\mathrm{N}_{4}\right.$ and $\left.\mathrm{Pt}-\mathrm{O}_{2}\right)$, the $\mathrm{SA} \mathrm{Pt}$ species coordinated with three $\mathrm{O}$ atoms $\left(\mathrm{Pt}-\mathrm{O}_{3}\right)$ display the highest turnover number value of $\mathbf{3 2 8 8}$ for the hydroboration reaction to access the important alkylboronic esters. Density functional theory calculations reveal that a superior catalytic activity can be expected for alkene hydroboration over the three $O$ coordinated $\mathrm{Pt}$ species due to the lowest reaction energy $(\Delta G)$ limiting step from the reaction phase diagram.
\end{abstract}

Keywords: single-atomic Pt catalyst, coordination structure, anti-Markovnikov hydroboration of alkenes, DFT calculations

\section{INTRODUCTION}

Single-atomic (SA) catalysts have recently attracted extensive attention due to their specific activity, ultrahigh atomic utilization, and uniform active sites [1-14]. In such catalysts, individual metal atoms are commonly isolated and stabilized by coordinated atoms from sup- ports [15-20]. Similar to homogenous catalysts, subtle differences in species and quantity of coordinated atoms will exert an important influence on the geometric and electronic structures of these metal atoms, which can further affect their catalytic activities [21-26]. Therefore, it is important to explore the relationship between the coordination structure and performance of SA catalysts, which has been scarcely investigated so far [27-29]. To deeply understand this relationship, clarifying the catalytic performances of a series of SA catalysts with different coordination structures is highly desirable and will be of great significance for guiding the synthesis of betterperforming SA catalysts.

Alkylboronic esters are key intermediates in organic synthesis for constructing various $\mathrm{C}-\mathrm{C}, \mathrm{C}-\mathrm{O}$, and $\mathrm{C}-\mathrm{N}$ bonds [30,31]. Catalytic hydroboration of alkenes with good regioselectivity is one of the direct and effective methods to synthesize such compounds [32,33]. Compared with traditional homogeneous catalysts for this reaction, the development of corresponding heterogeneous catalysts lags far behind, although they are more promising in practical applications because of the easy recovery of catalysts for reducing the cost and residual metal contamination [34]. Moreover, considering the copious waste in the reaction caused by the widely used boron source as bis(pinacolato)diboron $\left(\mathrm{B}_{2} \mathrm{Pin}_{2}\right)$ [35], the hydroboration with pinacolborane (HBPin) is more

\footnotetext{
${ }^{1}$ Department of Chemistry, Tsinghua University, Beijing 100084, China

${ }^{2}$ State Key Laboratory of Catalysis, Dalian Institute of Chemical Physics, Chinese Academy of Sciences, Dalian 116023, China

${ }^{3}$ Beijing Key Laboratory of Construction Tailorable Advanced Functional Materials and Green Applications, School of Materials Science and Engineering, Beijing Institute of Technology, Beijing 100081, China

${ }^{4}$ Beijing National Laboratory for Condensed Matter Physics, Institute of Physics, Chinese Academy of Sciences, Beijing 100190, China

${ }^{5}$ Beijing Synchrotron Radiation Facility, Institute of High Energy Physics, Chinese Academy of Sciences, Beijing 100049, China

† These authors contributed equally to this work.

* Corresponding author (email: wangdingsheng@mail.tsinghua.edu.cn)
} 
atom-economical and attractive but very challenging because of the low reactivity of HBPin [36-39]. Therefore, to realize the heterogeneous catalytic hydroboration of alkenes with HBPin as the boron source, it is crucial and imperative to prepare highly efficient heterogeneous catalysts.

Here, we demonstrate that the coordination structure of the SA Pt catalyst has a significant influence on its catalytic behavior for the anti-Markovnikov hydroboration of alkenes. Through different synthetic strategies, three SA Pt catalysts with $\mathrm{Pt}-\mathrm{O}_{3}, \mathrm{Pt}-\mathrm{O}_{2}$, and $\mathrm{Pt}-\mathrm{N}_{4}$ coordination structures are fabricated respectively. The $\mathrm{Pt}-\mathrm{O}_{3}$ structure of the SA Pt catalyst exhibits much higher activity for the hydroboration reaction than the other two structures $\left(\mathrm{Pt}-\mathrm{O}_{2}\right.$ and $\left.\mathrm{Pt}-\mathrm{N}_{4}\right)$, producing the important alkylboronic esters. Further density functional theory (DFT) calculations reveal that the superior performance of $\mathrm{Pt}-\mathrm{O}_{3}$ in $\mathrm{SA} \mathrm{Pt}$ catalyst is attributed to its adaptive reactive ability, namely suitable adsorption energies for all the adsorbates, which results in the lowest limiting reaction energy $(\Delta G)$.

\section{EXPERIMENTAL SECTION}

\section{Materials}

Iron nitrate nonahydrate $\left(\mathrm{Fe}\left(\mathrm{NO}_{3}\right)_{3} \cdot 9 \mathrm{H}_{2} \mathrm{O}, \mathrm{AR}\right)$, cobalt nitrate hexahydrate $\left(\mathrm{Co}\left(\mathrm{NO}_{3}\right)_{2} \cdot 6 \mathrm{H}_{2} \mathrm{O}, \mathrm{AR}\right)$, sodium borohydride $\left(\mathrm{NaBH}_{4}, 98.0 \%\right)$, cetyltrimethylammonium bromide (CTAB, AR), sodium hydroxide $(\mathrm{NaOH}, \mathrm{AR})$, sodium carbonate $\left(\mathrm{Na}_{2} \mathrm{CO}_{3}, 99.0 \%\right)$, hydrochloric acid ( $\mathrm{HCl}, 99.0 \%)$, ethylenediaminetetraacetic acid disodium salt (EDTA-2Na, 99.8\%) were purchased from Sinopharm Chemical Reagent Co. Ltd. (China). 1-Octene $\left(\mathrm{C}_{8} \mathrm{H}_{16}\right.$, 98.0\%), tetraammineplatinum(II) chloride monohydrate $\left(\mathrm{Cl}_{2} \mathrm{H}_{14} \mathrm{~N}_{4} \mathrm{OPt}, 99.9 \%\right)$, hexachloroplatinic acid $\left(\mathrm{H}_{2} \mathrm{PtCl}_{6}\right.$, 99.0\%) were provided by Alfa Asear (China). Titanium oxide $(99.8 \%, 5-10 \mathrm{~nm})$ was purchased from Aladdin (China). Pinacol borane was purchased from Energy Chemical (China). All reagents were used without further purification.

\section{Preparation of the catalysts}

\section{Synthesis of FeCo oxide nanosheets}

The FeCo oxide nanosheets (FeCo ONs) were prepared according to a method reported in previous reference [40] with some modifications. $1.0 \mathrm{mmol} \mathrm{Fe}\left(\mathrm{NO}_{3}\right)_{3} \cdot 9 \mathrm{H}_{2} \mathrm{O}$, $1.0 \mathrm{mmol} \mathrm{Co}\left(\mathrm{NO}_{3}\right)_{2} \cdot 6 \mathrm{H}_{2} \mathrm{O}$ and $0.5 \mathrm{~g} \mathrm{CTAB}$ were dispersed into $25 \mathrm{~mL}$ deionized water. After continuous stirring and ultrasonic treatment until all the solutes dissolved, freshly prepared $\mathrm{NaBH}_{4}$ solution $(0.1 \mathrm{~g}$ in $10 \mathrm{~mL}$ water) was added dropwise slowly. After the reaction proceeded for some time, the product was centrifuged and washed with ethanol three times and finally dried under vacuum overnight at room temperature.

Synthesis of $\mathrm{SA} \mathrm{Pt- \textrm {O } _ { 3 }}$

In a typical synthesis, $100 \mathrm{mg}$ as-prepared FeCo ONs was dispersed in $25 \mathrm{~mL}$ water under ultrasonic vibration. Afterwards, a $\mathrm{H}_{2} \mathrm{PtCl}_{6}$ solution ( $1.25 \mathrm{mg}$ in $10 \mathrm{~mL}$ water) was added dropwise under stirring at room temperature. After stirring for $12 \mathrm{~h}$, the mixed solution was centrifuged. The recovered solid was washed with deionized water and ethanol several times. After drying under vacuum overnight, the solid was transferred into a porcelain boat and then kept in a tube furnace maintaining at $100^{\circ} \mathrm{C}$ under $5 \% \mathrm{H}_{2} / \mathrm{N}_{2}$ atmosphere for $1 \mathrm{~h}$. Finally, the $\mathrm{SA} \mathrm{Pt}-\mathrm{O}_{3}$ was obtained.

\section{Synthesis of $\mathrm{SA} \mathrm{Pt- \textrm {O } _ { 2 }}$}

The SA Pt- $\mathrm{O}_{2}$ was prepared according to a previously reported method [41]. In a typical synthesis, the titanium oxide ( $1 \mathrm{~g}$ ) and an aqueous solution of sodium hydroxide $\left(10 \mathrm{~mol} \mathrm{~L}^{-1}, 30 \mathrm{~mL}\right)$ were stirred to form a suspension. Afterwards, the mixture was transferred into a $50-\mathrm{mL}$ Teflon-lined stainless-steel autoclave and hydrothermally treated at $130^{\circ} \mathrm{C}$ for $72 \mathrm{~h}$. After cooling down to room temperature, the precipitate was separated by centrifugation and washed with deionized water until a $\mathrm{pH}$ near 8 . The recovered solid was dried at $60^{\circ} \mathrm{C}$ for $12 \mathrm{~h}$ (marked as titanate nanotubes). Then, tetraammineplatinum(I) chloride monohydrate $(1.82 \mathrm{mg})$ was dissolved in an aqueous solution of $\mathrm{HCl}\left(0.1 \mathrm{~mol} \mathrm{~L}^{-1}\right.$, $20 \mathrm{~mL}$ ) to form a clarified solution. The as-prepared titanate nanotubes $(0.2 \mathrm{~g})$ were added to the above solution and mixed with ultrasonic treatment for $30 \mathrm{~min}$. The mixture was continuously stirred for $12 \mathrm{~h}$ at room temperature. The precipitate was separated by centrifugation at $10,000 \mathrm{r} \mathrm{min}^{-1}$ for $6 \mathrm{~min}$ and washed with deionized water for three times, then dried under vacuum at $60^{\circ} \mathrm{C}$ for $12 \mathrm{~h}$. After being ground, the as-prepared precipitate was calcined in a muffle furnace at $400^{\circ} \mathrm{C}$ for $1 \mathrm{~h}$ and then kept in a tube furnace at $160^{\circ} \mathrm{C}$ under the $5 \% \mathrm{H}_{2} / \mathrm{Ar}$ atmosphere for $1 \mathrm{~h}$ with a heating rate of $2^{\circ} \mathrm{C} \mathrm{min}{ }^{-1}$. Finally, the product, marked as SA Pt- $\mathrm{O}_{2}$, was collected without any treatment for further use.

Synthesis of SA Pt-N The SA Pt-N ${ }_{4}$ catalyst was synthesized based on previously reported work [42]. In a typical synthesis, $4 \mathrm{~g}$ 
EDTA-2Na and $36 \mathrm{mg} \mathrm{K}_{2} \mathrm{PtCl}_{6}$ were dissolved into $100 \mathrm{~mL}$ deionized water, and then stirred at $80^{\circ} \mathrm{C}$ for $12 \mathrm{~h}$. Afterwards, the solution was evaporated and transferred into a solid mixture of EDTA-2Na-Pt complex. The recovered solid was then thoroughly mixed with $40 \mathrm{~g} \mathrm{Na}_{2} \mathrm{CO}_{3}$ powder through grinding using an agate mortar. The mixed precursors were placed in a corundum boat and annealed at $850^{\circ} \mathrm{C}$ with a heating rate of $3^{\circ} \mathrm{C} \mathrm{min}{ }^{-1}$ under Ar atmosphere for $1 \mathrm{~h}$. After cooling down to room temperature, the resulting powder was transferred into a diluted hydrochloric acid solution to remove soluble $\mathrm{Na}_{2} \mathrm{CO}_{3}$ salt completely. Finally, the SA $\mathrm{Pt}-\mathrm{N}_{4}$ was collected by vacuum infiltration and then dried in oven overnight after washing with distilled water and anhydrous ethanol for several times.

\section{Catalytic evaluation}

All operations were performed with the use of standard Schlenk techniques. Unless otherwise noted, all the experiments were carried out under $\mathrm{N}_{2}$ atmosphere. In a typical procedure: 1-octene $(1.0 \mathrm{mmol})$, pinacol borane $(2.0 \mathrm{mmol})$ and the catalysts $\left(\mathrm{SA} \mathrm{Pt}-\mathrm{O}_{3}, \mathrm{SA} \mathrm{Pt}-\mathrm{O}_{2}, \mathrm{SA}\right.$ $\mathrm{Pt}-\mathrm{N}_{4}, \mathrm{Pt} /$ substrate$=0.028 \%$ ) were placed in a Schlenk tube. The reactor was sealed and purged three times with $\mathrm{N}_{2}$ at $1 \mathrm{MPa}$. Then the mixture was heated at $40^{\circ} \mathrm{C}$ under vigorously stirring $\left(800 \mathrm{rmin}^{-1}\right)$ for $24 \mathrm{~h}$. After the reaction, the yield was detected by gas chromatography (GC) using dodecane as the internal standard. The overall calculated turnover number $(\mathrm{TON},=($ mole of product 3$) /$ (mole of $\mathrm{Pt}$ )) was measured upon completion of reactions and the calculation of it was based on the total Pt loading in the catalysts.

\section{Characterization}

X-ray diffraction (XRD) data were obtained from a Rigaku D/max 2500Pc X-ray powder diffractometer (Japan) using a $\mathrm{Cu} \mathrm{Ka}$ radiation $(\lambda=0.15418 \mathrm{~nm})$. Transmission electron microscopy (TEM) images were taken on a $\mathrm{Hi}$ tachi-7700 (Japan) operated at $100 \mathrm{kV}$. High resolution TEM (HRTEM) images were recorded on a JEOL JEM$2100 \mathrm{~F}$ field emission transmission electron microscope (Japan) operated at $200 \mathrm{kV}$. Aberration-corrected highangle annular dark-field scanning transmission electron microscopy (AC HAADF-STEM) images were taken on a Titan 80-300 scanning/transmission electron microscope (FEI, USA) operated at $300 \mathrm{kV}$, equipped with a probe spherical aberration corrector. Inductively coupled plasma optical emission spectrometry (ICP-OES) measurements were carried out on a Thermo Fisher iCAP ${ }^{\mathrm{TM}} 7000$ Series ICP-OES analyzer (USA). The product was iden- tified by gas chromatography-mass spectrometry (GCMS, Thermo Fisher Scientific-TXQ Quntum XLS, USA), and was quantitatively analyzed by GC (Shimadzu, GC2010 Plus, Japan), equipped with FID using a capillary column (TR-5MS, from Thermo Scientific, USA; length $30 \mathrm{~m}$, inner diameter $0.25 \mathrm{~mm}$, film $0.25 \mu \mathrm{m}$ ) employing $n$-octanol as an internal standard. ${ }^{1} \mathrm{H}$ and ${ }^{13} \mathrm{C}$ NMR data were acquired from a Bruker Advance III $(400 \mathrm{MHz})$ spectrometer (Germany).

\section{XAFS analysis and results}

The X-ray absorption find structure (XAFS) data of the sample were collected at 1W1B station in Beijing Synchrotron Radiation Facility (BSRF). The acquired extended XAFS (EXAFS) data were processed according to the standard procedures using the ATHENA module implemented in the IFEFFIT software packages. The EXAFS spectra were obtained by subtracting the postedge background from the overall absorption and then normalizing with respect to the edge-jump step. Subsequently, $k^{3}$-weighted $\chi(k)$ data of $\mathrm{Pt} \mathrm{L}_{3}$-edge was Fourier transformed to real $(\mathrm{R})$ space using a hanning windows ( $\mathrm{d} k=1.0 \AA^{-1}$ ) to separate the EXAFS contributions from different coordination shells. To obtain the quantitative structural parameters around central atoms, least-squares curve parameter fitting was performed using the ARTEMIS module of IFEFFIT software packages. The following EXAFS equation was used:

$$
\begin{aligned}
\chi(k)= & \sum_{j} \frac{N_{j} S_{0}^{2} F_{j}(k)}{k R_{j}^{2}} \exp \left[-2 k^{2} \sigma_{j}^{2}\right] \\
& \times \exp \left[\frac{-2 R_{j}}{\lambda(k)}\right] \sin \left[2 k R_{j}+\phi_{j}(k)\right],
\end{aligned}
$$

where $S_{0}{ }^{2}$ is the amplitude reduction factor, $F_{j}(k)$ is the effective curved-wave backscattering amplitude, $N_{j}$ is the number of neighbors in the $j^{\text {th }}$ atomic shell, $R_{j}$ is the distance between the X-ray absorbing central atom and the atoms in the $j^{\text {th }}$ atomic shell (backscatterer), $\lambda$ is the mean free path in $\AA, \phi_{j}(k)$ is the phase shift (including the phase shift for each shell and the total central atom phase shift), $\sigma_{j}$ is the Debye-Waller parameter of the $j^{\text {th }}$ atomic shell (variation of distances around the average $R_{j}$ ). The functions $F_{j}(k), \lambda$ and $\phi_{j}(k)$ were calculated with the $a b$ initio code FEFF8.2.

\section{Computational method}

The DFT calculations were conducted using the Vienna $A b$ initio Simulation Package (VASP) $[43,44]$ applying the functional of revised Perdew-Burke-Ernzerhof (rPBE) 
$[45,46]$ and generalized gradient approximation (GGA). The interactions between electrons and atomic cores were described using the projector augmented wave (PAW) method. All the structure optimizations were conducted with a cut-off energy of $400 \mathrm{eV}$ and the force convergency of $0.05 \mathrm{eV}^{-1}$. The transition states were obtained using the climbing image nudged elastic band (NEB) [47] with a same force convergency of $0.05 \mathrm{eV} \AA^{-1}$. All the adsorption energies were refered to the gas energies of octene $\left(\mathrm{C}_{8} \mathrm{H}_{16}\right)$, hydrogen $\left(\mathrm{H}_{2}\right)$ and HBPin. The standard thermodynamic corrections $[48,49]$ were conducted to correct all the energies to free energy with the condition of the temperature of $313 \mathrm{~K}$ (correction values see Table S1).

Single atom Pt carried by three different catalysts were studied in this work, including $\mathrm{TiO}_{2}\left(\mathrm{SA} \mathrm{Pt}-\mathrm{O}_{2}\right), \mathrm{N}$-doped graphene $\left(\mathrm{SA} \mathrm{Pt}-\mathrm{N}_{4}\right)$ and FeCo oxide $\left(\mathrm{SA} \mathrm{Pt}-\mathrm{O}_{3}\right)$. The anatase $\mathrm{TiO}_{2}$ was used as a bulk structure, where a $\mathrm{TiO}_{2}$ (101) surface was applied with a $\mathrm{p}(2 \times 2)$ unit cell. A Monkhorst-Pack $k$-point $1 \times 3 \times 1$ was used in all the surface structure calculations for $\mathrm{TiO}_{2}(101)$. A p $(6 \times 6)$ graphene surface was applied with 4 doping N. A Monkhorst-Pack $k$-point $2 \times 2 \times 1$ was used in all the surface structure calculations for graphene. The spinel bulk structure $\mathrm{CoFe}_{2} \mathrm{O}_{4}$ was used to build the surface of FeCo oxide, where a $\mathrm{CoFe}_{2} \mathrm{O}_{4}(311)$ surface was used with a $\mathrm{p}(1 \times 1)$ unit cell. A Monkhorst-Pack $k$-point $1 \times 3 \times 1$ was used in all the surface structure calculations for $\mathrm{CoFe}_{2} \mathrm{O}_{4}$ (311).

Five different key adsorbates (ad-1 to ad-5) were considered based on the catalytic system (Fig. S1a), where the linear relations, namely the scaling relations (Fig. S2), were calculated with the descriptor of the adsorption energies of $\mathrm{C} 8 \mathrm{H} 17^{\star}$ (ad-2). Six elementary reactions (Fig. S1b) were considered in this work to establish the reaction phase diagram.

\section{RESULTS AND DISCUSSION}

The TEM image illustrates the hierarchical microstructure and uniform thickness of the as-prepared FeCo ONs, and the exclusive observed weak peak in the XRD pattern reveals the low crystalline of the sample (Figs S3 and S4). TEM and HAADF-STEM images indicate that the hierarchical microstructure is preserved after the adsorption of Pt element and simultaneously no visible Pt nanoparticles formed on the FeCo ONs support (Fig. 1a, b). No extra peaks consistent with Pt nanoparticles are found in the XRD pattern as well (Fig. S4). Energy-dispersive X-ray spectroscopy (EDS) shows that the Pt element is homogeneously dispersed on the support (Fig. 1c). The loading of Pt element is measured up to 0.54 weight percent ( $\mathrm{wt} \%$ ) according to the analysis of ICP-OES. To further confirm the sole existence of $\mathrm{Pt}$ species, we performed the AC HAADF-STEM measure-
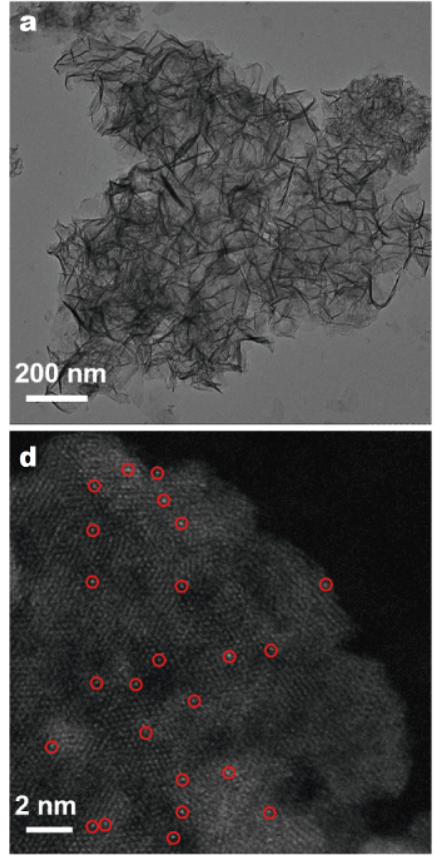
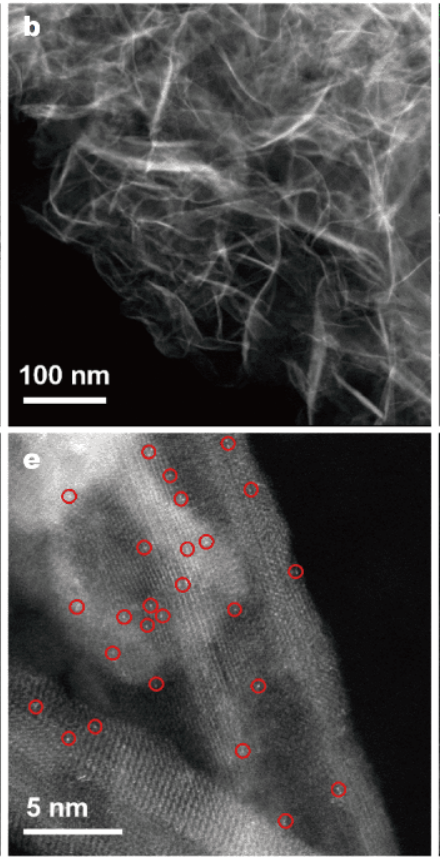
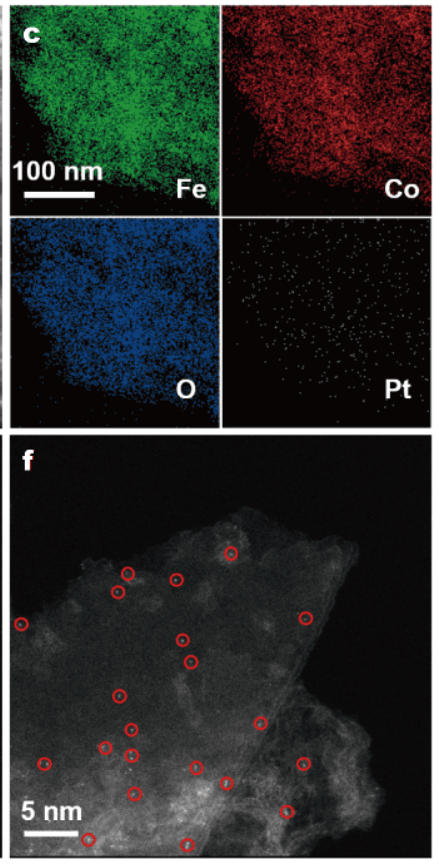

Figure 1 (a) TEM image, and (b) HAADF-STEM image of the SA Pt- $\mathrm{O}_{3}$ sample. (c) Corresponding EDS elemental maps of Fe, Co, O and Pt. (d) AC HAADF-STEM image of the SA Pt-O $\mathrm{O}_{3}$ sample. (e, f) AC HAADF-STEM images of the SA Pt- $\mathrm{O}_{2}$ and SA Pt- $\mathrm{N}_{4}$. 
ments on the catalyst (Fig. 1d). It can be recognized that there are only isolated bright dots corresponding to the single $\mathrm{Pt}$ atoms appearing on the $\mathrm{FeCo} \mathrm{ONs}$, demonstrating the sole presence of SA Pt species in the SA $\mathrm{Pt}-\mathrm{O}_{3}$.

To get more information about the electronic and coordination structures of the SA Pt catalyst, XAFS analysis was conducted. Fig. $2 \mathrm{~b}$ shows the Fourier transformed EXAFS (FT-EXAFS) spectrum of SA Pt- $\mathrm{O}_{3}$ with Pt-foil and $\mathrm{PtO}_{2}$ as references at the $\mathrm{Pt}_{3}$-edge. There is only one prominent peak at $1.6 \AA$ which can be attributed to the $\mathrm{Pt}-\mathrm{O}$ contribution and no peak corresponding to $\mathrm{Pt}-\mathrm{Pt}$ contribution at $2.6 \AA$ can be observed, confirming that $\mathrm{Pt}$ species exist as isolated atoms stabilized by oxygen atoms. Besides, the oxidation state of these SA Pt species is also illustrated by the X-ray absorption near-edge structure (XANES) spectra (Fig. 2a). In general, the white line intensities are closely related to the oxidation state of Pt. In Fig. 2a, the white line intensity of SA Pt- $\mathrm{O}_{3}$ is located between that of $\mathrm{Pt}$ foil and $\mathrm{PtO}_{2}$, which indicates that the oxidation state of $\mathrm{Pt}$ in $\mathrm{SA} \mathrm{Pt}-\mathrm{O}_{3}$ is situated between 0 and +4 . Further fitting analysis of the FT-EXAFS curve in R space confirmes that the SA Pt species are coordinated by three $\mathrm{O}$ atoms with a Pt-O bond distance of $1.97 \AA$ to form the Pt- $\mathrm{O}_{3}$ structure (Fig. 2c and Table S2). The local structure of Pt species obtained from DFT simulations is displayed in Fig. 2d.

The other two SA Pt catalysts with $\mathrm{Pt}-\mathrm{O}_{2}$ and $\mathrm{Pt}-\mathrm{N}_{4}$ coordination structures (denoted as SA Pt- $\mathrm{O}_{2}$ and SA Pt$\mathrm{N}_{4}$ ) were synthesized according to different strategies $[41,42]$. As revealed by TEM images (Figs S5 and S6) and XRD patterns (Figs S7 and S8), there are no obvious peaks matching with Pt nanoparticles. EDS demonstrates that all elements are uniformly dispersed (Figs S9 and S10). The contents of Pt element were separately measured up to $0.51 \mathrm{wt} \%\left(\mathrm{SA} \mathrm{Pt}-\mathrm{O}_{2}\right)$ and $0.56 \mathrm{wt} \%\left(\mathrm{SA} \mathrm{Pt}-\mathrm{N}_{4}\right)$ by ICP-OES. Besides, the HAADF-STEM (Figs S11 and S12) and AC HAADF-STEM images (Fig. 1e, f) indicate that Pt element exists in isolated single atoms for both samples, which confirms the successful syntheses of the other two SA Pt catalysts.

We next evaluated the catalytic performance of SA $\mathrm{Pt}_{-} \mathrm{O}_{3}$ for the hydroboration of alkenes (Fig. 3). Initially, the hydroboration of 1-octene with the relatively inert HBPin (1) as the boron source was chosen as the model reaction. It was delighting that 1 -octene (2) was almost
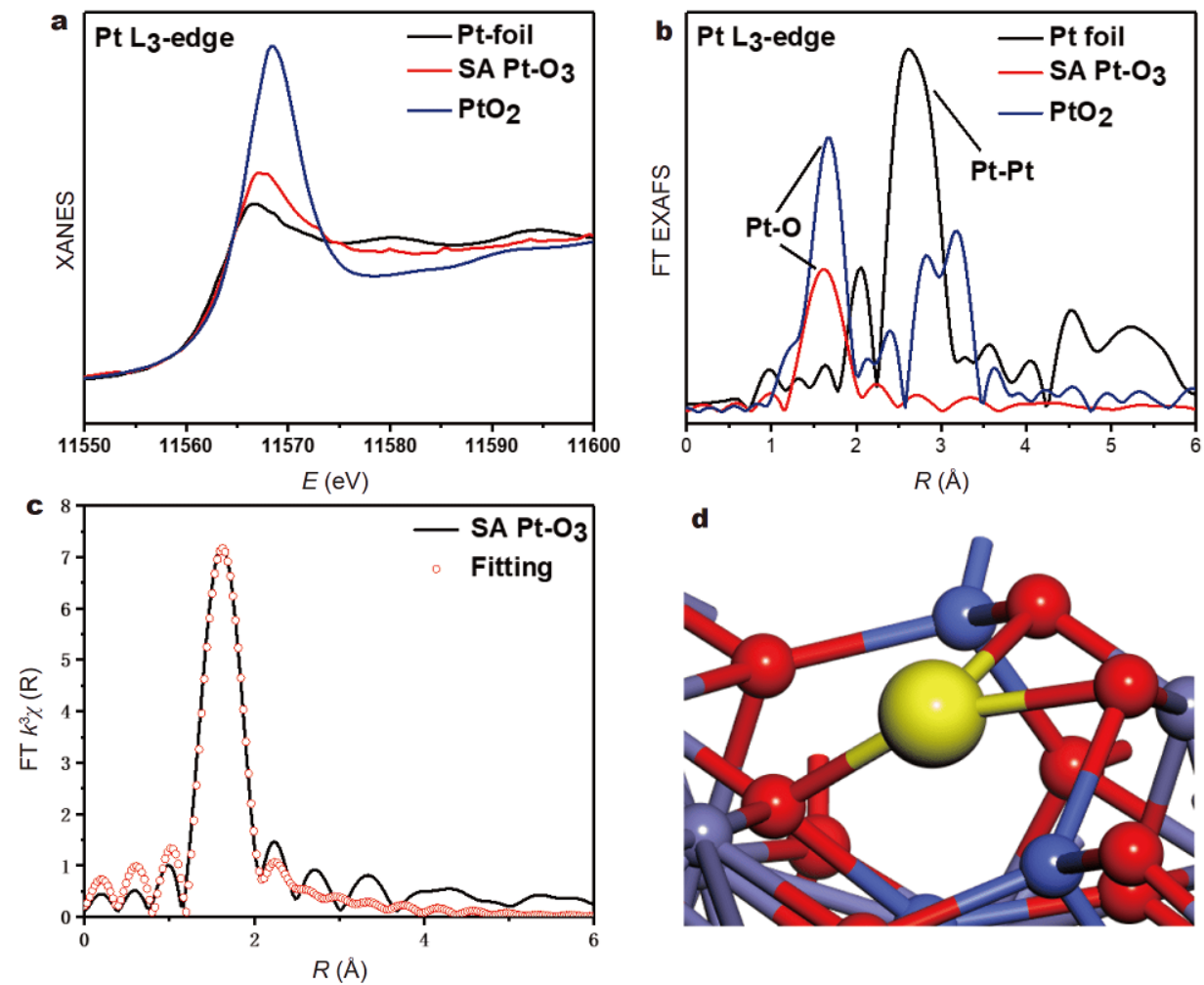

Figure 2 (a) XANES spectra at the $\mathrm{Pt}_{3}$-edge of the SA Pt- $\mathrm{O}_{3}$ sample, $\mathrm{PtO}_{2}$, and Pt foil. (b) FT-EXAFS spectra at the Pt $\mathrm{L}_{3}$-edge of the SA Pt- $\mathrm{O}_{3}$ sample, $\mathrm{PtO}_{2}$, and Pt foil. (c) Corresponding fits of the EXAFS spectrum of the SA Pt- $\mathrm{O}_{3}$ at the R space. (d) Local structure of Pt species in SA Pt-O from DFT simulations. The yellow, red, blue and purple balls represent Pt, O, Co and Fe atoms, respectively. 


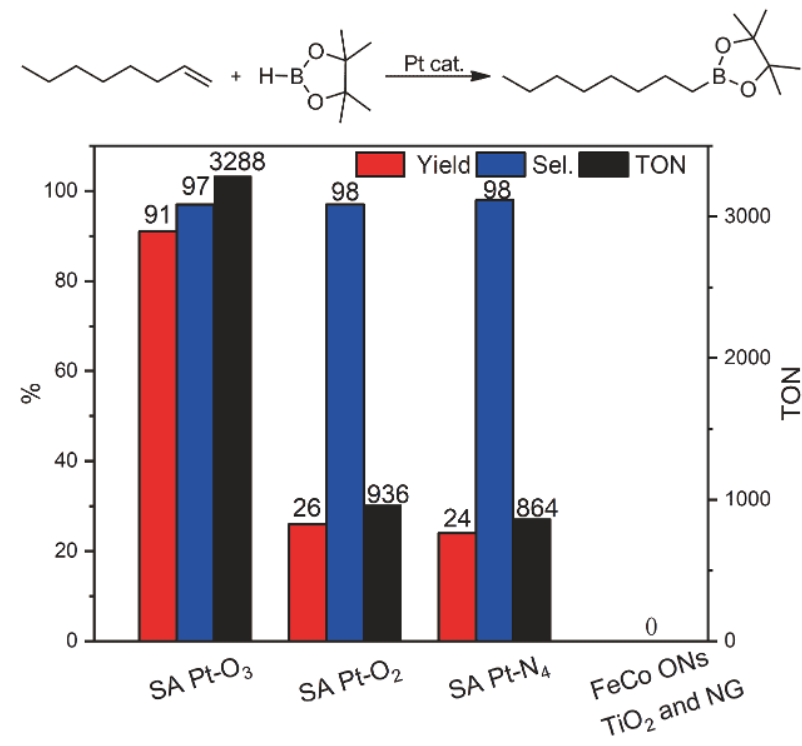

Figure 3 A performance comparison of SA Pt- $\mathrm{O}_{3}, \mathrm{SA} \mathrm{Pt}-\mathrm{O}_{2}, \mathrm{SA} \mathrm{Pt}-\mathrm{N}_{4}$, and pure supports in anti-Markovnikov hydroboration of 1-octene.

completely converted to the target anti-Markovnikov addition alkylboronic ester (3) (Figs S13 and S14) in 91\% yield and $97 \%$ selectivity over the $\mathrm{SA} \mathrm{Pt-O}$ without significant alkene isomerization. The TON value of SA Pt$\mathrm{O}_{3}$ was up to 3288. As for the other two SA Pt catalysts, the yield of the product 3 dropped down to $26 \%$ for SA $\mathrm{Pt}-\mathrm{O}_{2}$ and $24 \%$ for SA Pt- $\mathrm{N}_{4}$ accompanied by a certain degree of alkene isomerization, achieving the much lower TON values as 936 and 864, respectively. In contrast, almost no product was obtained when the pure FeCo ONs, $\mathrm{TiO}_{2}$ and $\mathrm{N}$-doped graphene (NG) were used as the catalysts. These results of control experiments demonstrate that the catalytic active species is SA Pt and the different performances of the three catalysts for the hydroboration reaction are probably concerned with the distinct coordination structures of $\mathrm{Pt}$ atoms. After the reaction, according to HAADF-STEM, AC HAADFSTEM images and XRD pattern, the initial morphology of SA Pt- $\mathrm{O}_{3}$ remained unchanged and no agglomeration of Pt species was found after the reaction (Figs S15 and S16).

Towards further understanding the relationship between the coordination structure and the catalytic performance of SA Pt-O $\mathrm{O}_{3}$, DFT studies were conducted to gain more insight into the catalytic mechanism and activity trend of the hydroboration reaction over the above three catalysts with different coordination structures (Fig. S17). Five different key adsorbates (ad-1 to ad-5) were considered based on the catalytic systems (Fig. S1a), where linear relations (Fig. S2), namely the scaling rela- tions [50,51], were calculated with the descriptor of the adsorption energies of $\mathrm{C}_{8} \mathrm{H}_{17}$ (ad-2). Six elementary reactions (Fig. S1b) were considered in this work to establish the reaction phase diagram (RPD) [52,53] (Fig. 4a).

Two different reaction pathways were considered in this work, which can be mainly dependent on the different priorities of the first activation of ad-1 by hydrogenation (Fig. 4b, Pathway 2) or hydroboration (Fig. 4b, Pathway 1). The Pathway 1 is more favored, when the adsorption energy of ad-2, namely the descriptor (described in Fig. $4 \mathrm{a}, X$-axis), is lower than $-0.75 \mathrm{eV}$ due to a higher reaction energy of R5 needed for Pathway 2. With the increase of the ad-2 adsorption energy, a higher reaction energy of R2 can be found, resulting in a difficult reaction by Pathway 1 . Therefore, Pathway 2 is more favored in this case.

The $\Delta G$ limiting steps can be obtained as the elementary step with the highest reaction energy in specific reaction pathways. Here, different $\Delta G$ limiting steps are demonstrated for alkene hydroboration with different adsorption energies of ad-2. Importantly, the catalytic activity can be described from the reaction energy of $\Delta G$ limiting steps. It is found that the reaction energy of $\Delta G$ limiting step for alkene hydroboration over $\mathrm{SA} \mathrm{Pt}-\mathrm{O}_{3}$ is just located close to the lowest reaction energy obtained from RPD, which is expected to show the highest catalytic activity (Fig. 4a). Hereafter, a theoretically predicted activity trend can be obtained to be SA Pt- $\mathrm{O}_{3}>\mathrm{SA}$ Pt- $\mathrm{O}_{2}>\mathrm{SA}$ Pt- $\mathrm{N}_{4}$, which shows an excellent agreement compared with the experimental results discussed above. Note that, although the scaling relations show a general trend for the adsorption energies between different adsorbates, it may affect the predicted activity trend due to the error in the linear fitiing compared to the explicit calculation. Accordindly, The activation barriers of $\Delta G$ limiting steps were calculated to further prove the expected catalytic activity for SA Pt- $\mathrm{O}_{3}$. R6 is obtained to be the $\Delta G$ limiting step for alkene hydroboration over $\mathrm{SA} \mathrm{Pt}-\mathrm{O}_{2}$, where a higher activation barrier of $1.47 \mathrm{eV}$ is calculated on SA $\mathrm{Pt}-\mathrm{O}_{2}$ compared with that of only $0.09 \mathrm{eV}$ on SA Pt- $\mathrm{O}_{3}$. Meanwhile, the dissociative adsorption of HBPin (R1) is found to be the $\Delta G$ limiting step on SA Pt- $\mathrm{N}_{4}$ with a higher activation barrier of $1.91 \mathrm{eV}$, while $0.47 \mathrm{eV}$ of activation barrier on $\mathrm{SA} \mathrm{Pt}-\mathrm{O}_{3}$ is calculated.

As discussed above, the alkene hydroboration over SA $\mathrm{Pt}-\mathrm{O}_{3}$ is just located close to the point with the lowest $\Delta G$ limiting reaction energy. Seriously, it is difficult to judge a clear reaction mechanism for alkene hydroboration over SA Pt- $\mathrm{O}_{3}$ due to a similar energy of $\Delta G$ limiting reaction 
energy for different pathways, where it is located just at the switch point for different pathways based on the reaction phase diagram. Therefore, further DFT calcula- tions were conducted to investigate the reaction mechanism for alkene hydroboration over SA $\mathrm{Pt}_{-} \mathrm{O}_{3}$ (Fig. 5a) with the calculations of all the transition states
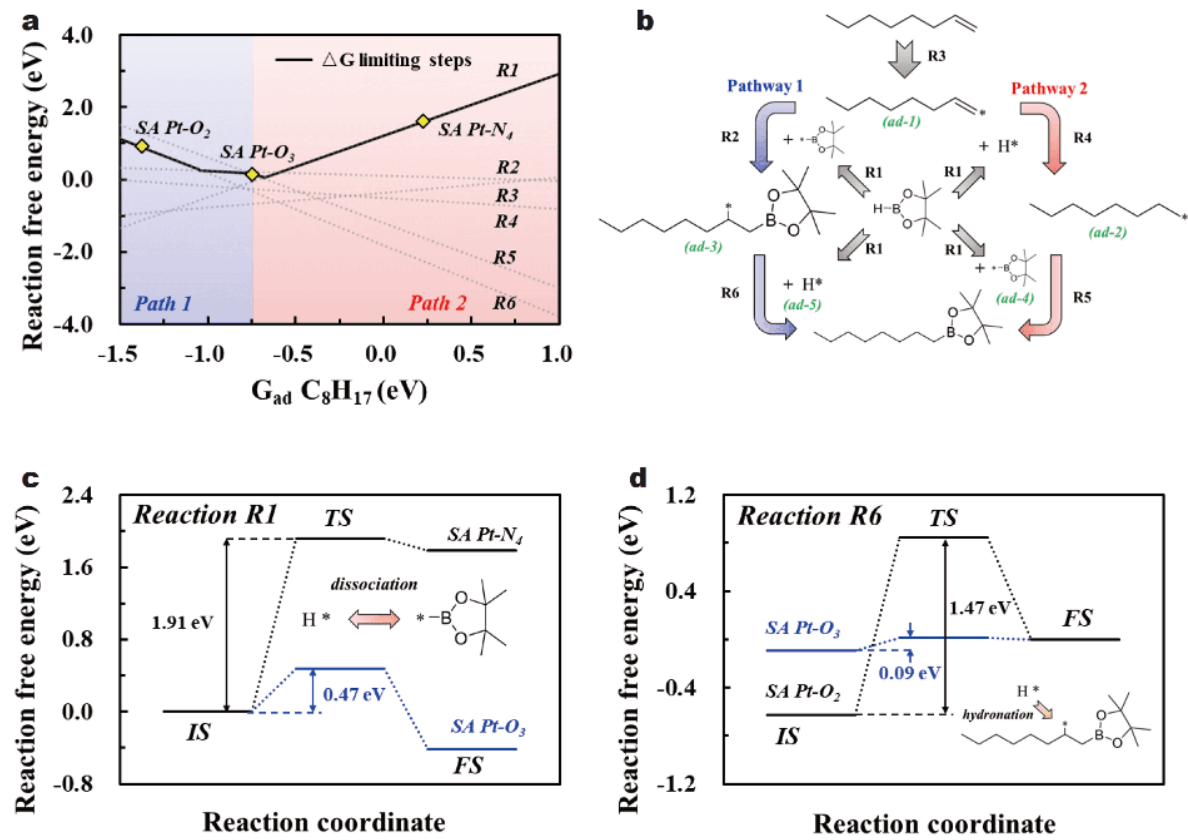

Figure 4 (a) Reaction phase diagram of alkene hydroboration (elementary steps R1-R6 see the Supplementary information). The black solid lines are $\Delta G$ limiting steps; the blue and red backgrounds refer two different reaction pathways. (b) Two different reaction pathways for alkene hydroboration. (c) Activation barriers of R1 over SA Pt- $\mathrm{O}_{3}$ and SA Pt- $\mathrm{N}_{4}$. (d) Activation barriers of R6 over SA Pt- $\mathrm{O}_{3}$ and SA Pt- $\mathrm{O}_{2}$.

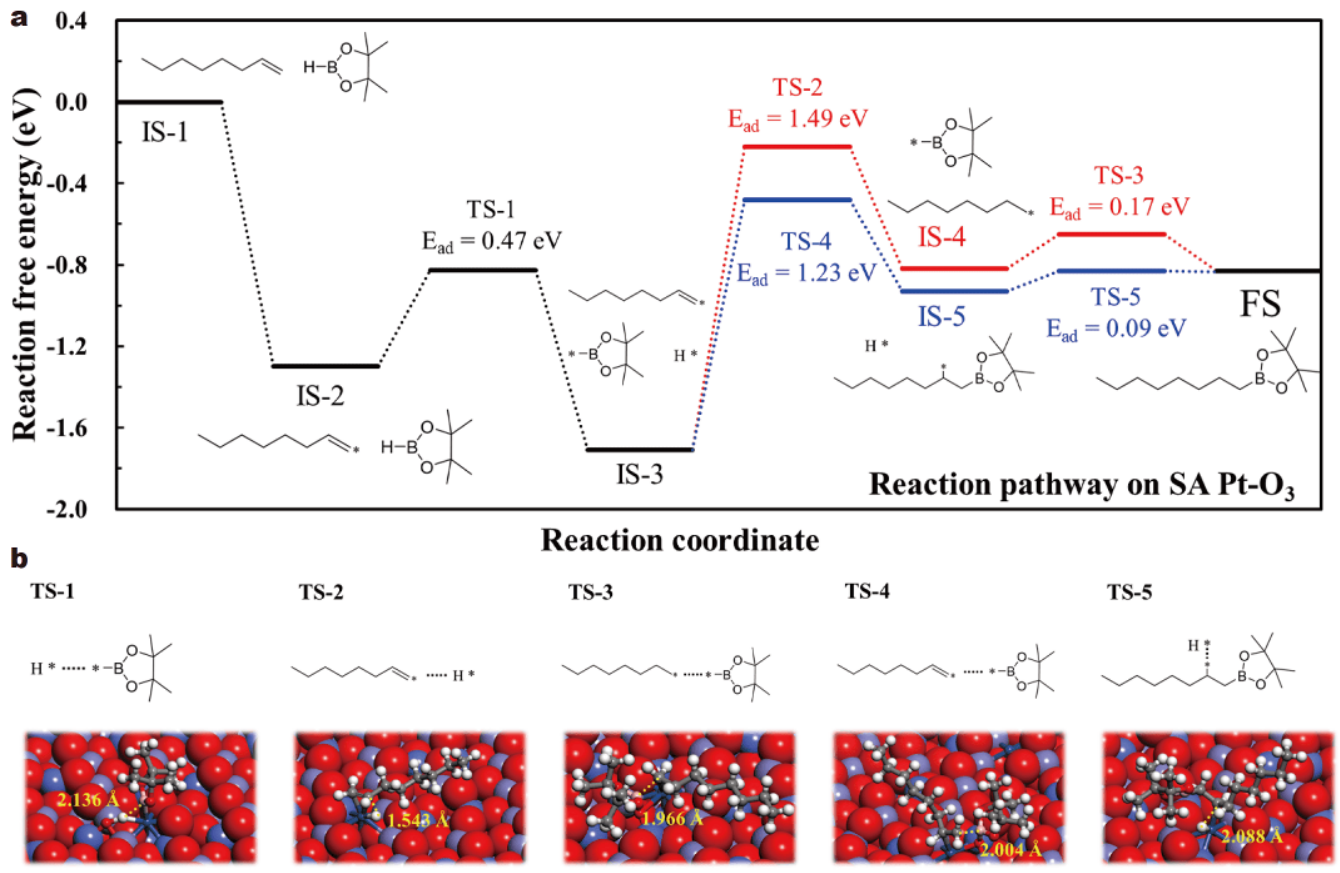

Figure 5 (a) Reaction pathways of alkene hydroboration over SA Pt- ${ }_{3}$. The red and blue lines show different pathways. (b) Transition state structures performed in alkene hydroboration over SA Pt- $\mathrm{O}_{3}$. 
(Fig. 5b).

It is found that lower activation barrier of $1.23 \mathrm{eV}$ is obtained for the hydroboration of $\mathrm{C}_{8} \mathrm{H}_{17}$ * compared with the hydrogenation $(1.49 \mathrm{eV})$. Meanwhile, the following steps of both pathways show lower activation barrier affecting less the total activity. As a result, the mechanism with the hydroboration of $\mathrm{C}_{8} \mathrm{H}_{17}$ * followed by a further hydrogenation is more favored over $\mathrm{SA} P \mathrm{Pt}-\mathrm{O}_{3}$. Additionally, the better activation behavior for alkene hydroboration over SA Pt- $\mathrm{O}_{3}$ can be further proved with the lower activation barrier of $\Delta G$ limiting barrier $(1.23 \mathrm{eV})$ compared with that on either SA Pt- $\mathrm{O}_{2}(1.47 \mathrm{eV}$, Fig. 4c) or SA Pt- $\mathrm{N}_{4}(1.91 \mathrm{eV}$, Fig. $4 \mathrm{~d})$.

\section{CONCLUSIONS}

In summary, the influence of the coordination structure on the catalytic properties of SA catalysts has been thoroughly investigated by considering three SA Pt catalysts: SA Pt- $\mathrm{O}_{3}$, SA Pt- $\mathrm{O}_{2}$, and SA Pt- $\mathrm{N}_{4}$. The as-fabricated SA Pt- $\mathrm{O}_{3}$ exhibits the highest activity in the antiMarkovnikov hydroboration of alkenes, producing the valuable alkylboronic esters with good selectivity. As revealed by DFT calculation and experimental results, the superior performance of SA Pt- $\mathrm{O}_{3}$ is attributed to its distinctive coordination structure, which results in the lowest reaction energy of $\Delta G$ limiting step. This work may provide new insights for preparing novel betterperforming SA catalysts by changing the coordination structure of the active metal centers.

Received 27 March 2020; accepted 3 April 2020;

published online 15 May 2020

1 Chen Z, Vorobyeva E, Mitchell S, et al. A heterogeneous singleatom palladium catalyst surpassing homogeneous systems for $\mathrm{Su}$ zuki coupling. Nat Nanotech, 2018, 13: 702-707

2 Cui X, Li W, Ryabchuk P, et al. Bridging homogeneous and heterogeneous catalysis by heterogeneous single-metal-site catalysts. Nat Catal, 2018, 1: 385-397

$3 \mathrm{Gu}$ J, Hsu CS, Bai L, et al. Atomically dispersed $\mathrm{Fe}^{3+}$ sites catalyze efficient $\mathrm{CO}_{2}$ electroreduction to CO. Science, 2019, 364: 10911094

4 Liu L, Corma A. Metal catalysts for heterogeneous catalysis: from single atoms to nanoclusters and nanoparticles. Chem Rev, 2018, 118: 4981-5079

5 Liu P, Zhao Y, Qin R, et al. Photochemical route for synthesizing atomically dispersed palladium catalysts. Science, 2016, 352: 797800

6 Matsubu JC, Yang VN, Christopher P. Isolated metal active site concentration and stability control catalytic $\mathrm{CO}_{2}$ reduction selectivity. J Am Chem Soc, 2015, 137: 3076-3084

7 Sun T, Xu L, Wang D, et al. Metal organic frameworks derived single atom catalysts for electrocatalytic energy conversion. Nano Res, 2019, 12: 2067-2080
8 Yang F, Ding S, Song H, et al. Single-atom Pd dispersed on nanoscale anatase $\mathrm{TiO}_{2}$ for the selective hydrogenation of phenylacetylene. Sci China Mater, 2020, 63: doi: 10.1007/s40843-0201271-x

9 Chen LN, Hou KP, Liu YS, et al. Efficient hydrogen production from methanol using a single-site $\mathrm{Pt}_{1} / \mathrm{CeO}_{2}$ catalyst. J Am Chem Soc, 2019, 141: 17995-17999

10 Zhao L, Zhang Y, Huang LB, et al. Cascade anchoring strategy for general mass production of high-loading single-atomic metalnitrogen catalysts. Nat Commun, 2019, 10: 1278

11 Feng Y, Zhou L, Wan Q, et al. Selective hydrogenation of 1,3butadiene catalyzed by a single Pd atom anchored on graphene: the importance of dynamics. Chem Sci, 2018, 9: 5890-5896

12 Fang X, Shang Q, Wang Y, et al. Single Pt atoms confined into a metal-organic framework for efficient photocatalysis. Adv Mater, 2018, 30: 1705112

13 Jiao L, Jiang HL. Metal-organic-framework-based single-atom catalysts for energy applications. Chem, 2019, 5: 786-804

14 Xiong $\mathrm{Y}$, Dong J, Huang ZQ, et al. Single-atom Rh/N-doped carbon electrocatalyst for formic acid oxidation. Nat Nanotechnol, 2020, 15: doi: 10.1038/s41565-020-0665-x

15 Yamada Y, Tsung CK, Huang W, et al. Nanocrystal bilayer for tandem catalysis. Nat Chem, 2011, 3: 372-376

16 Deng D, Chen X, Yu L, et al. A single iron site confined in a graphene matrix for the catalytic oxidation of benzene at room temperature. Sci Adv, 2015, 1: e1500462

17 Li S, Xu Y, Chen Y, et al. Tuning the selectivity of catalytic carbon dioxide hydrogenation over iridium/cerium oxide catalysts with a strong metal-support interaction. Angew Chem Int Ed, 2017, 56: 10761-10765

18 Bayatsarmadi B, Zheng Y, Vasileff A, et al. Recent advances in atomic metal doping of carbon-based nanomaterials for energy conversion. Small, 2017, 13: 1700191

19 Liu W, Zhang L, Yan W, et al. Single-atom dispersed Co-N-C catalyst: structure identification and performance for hydrogenative coupling of nitroarenes. Chem Sci, 2016, 7: 5758-5764

20 Li H, Cao C, Liu J, et al. Cobalt single atoms anchored on N-doped ultrathin carbon nanosheets for selective transfer hydrogenation of nitroarenes. Sci China Mater, 2019, 62: 1306-1314

$21 \mathrm{Du} \mathrm{Z}, \mathrm{Chen} \mathrm{X}, \mathrm{Hu} \mathrm{W}$, et al. Cobalt in nitrogen-doped graphene as single-atom catalyst for high-sulfur content lithium-sulfur batteries. J Am Chem Soc, 2019, 141: 3977-3985

22 Wang X, Chen Z, Zhao X, et al. Regulation of coordination number over single Co sites: triggering the efficient electroreduction of $\mathrm{CO}_{2}$. Angew Chem Int Ed, 2018, 57: 1944-1948

23 Yin XP, Wang HJ, Tang SF, et al. Engineering the coordination environment of single-atom platinum anchored on graphdiyne for optimizing electrocatalytic hydrogen evolution. Angew Chem Int Ed, 2018, 57: 9382-9386

24 Jiang $\mathrm{R}, \mathrm{Li} \mathrm{L}$, Sheng $\mathrm{T}$, et al. Edge-site engineering of atomically dispersed $\mathrm{Fe}-\mathrm{N}_{4}$ by selective $\mathrm{C}-\mathrm{N}$ bond cleavage for enhanced oxygen reduction reaction activities. J Am Chem Soc, 2018, 140: 11594-11598

25 Sun $\mathrm{Q}$, Wang $\mathrm{N}$, Zhang $\mathrm{T}$, et al. Zeolite-encaged single-atom rhodium catalysts: highly-efficient hydrogen generation and shapeselective tandem hydrogenation of nitroarenes. Angew Chem Int Ed, 2019, 58: 18570-18576

26 Li X, Rong $\mathrm{H}$, Zhang J, et al. Modulating the local coordination environment of single-atom catalysts for enhanced catalytic performance. Nano Res, 2020, 13: doi: 10.1007/s12274-020-2755-3 
27 Fei H, Dong J, Feng Y, et al. General synthesis and definitive structural identification of $\mathrm{MN}_{4} \mathrm{C}_{4}$ single-atom catalysts with tunable electrocatalytic activities. Nat Catal, 2018, 1: 63-72

28 Liu W, Zhang L, Liu X, et al. Discriminating catalytically active $\mathrm{FeN}_{x}$ species of atomically dispersed $\mathrm{Fe}-\mathrm{N}-\mathrm{C}$ catalyst for selective oxidation of the C-H bond. J Am Chem Soc, 2017, 139: 1079010798

29 Pan Y, Zhang C, Lin Y, et al. Electrocatalyst engineering and structure-activity relationship in hydrogen evolution reaction: from nanostructures to single atoms. Sci China Mater, 2020, 63: doi: 10.1007/s40843-019-1242-1

30 Frisch AC, Beller M. Catalysts for cross-coupling reactions with non-activated alkyl halides. Angew Chem Int Ed, 2005, 44: 674688

31 Mlynarski SN, Schuster CH, Morken JP. Asymmetric synthesis from terminal alkenes by cascades of diboration and crosscoupling. Nature, 2014, 505: 386-390

32 Coombs JR, Haeffner F, Kliman LT, et al. Scope and mechanism of the Pt-catalyzed enantioselective diboration of monosubstituted alkenes. J Am Chem Soc, 2013, 135: 11222-11231

33 Kamei T, Nishino S, Shimada T. Ni-catalyzed hydroboration and hydrosilylation of olefins with diboron and silylborane. Tetrahedron Lett, 2018, 59: 2896-2899

34 Ji P, Sawano T, Lin Z, et al. Cerium-hydride secondary building units in a porous metal-organic framework for catalytic hydroboration and hydrophosphination. J Am Chem Soc, 2016, 138: $14860-14863$

35 Park J, Lee Y, Kim J, et al. Copper-catalyzed diastereoselective addition of diborylmethane to $N$-tert-butanesulfinyl aldimines: synthesis of $\beta$-aminoboronates. Org Lett, 2016, 18: 1210-1213

36 Kisan S, Krishnakumar V, Gunanathan C. Ruthenium-catalyzed anti-markovnikov selective hydroboration of olefins. ACS Catal, 2017, 7: 5950-5954

37 Zhang L, Zuo Z, Leng X, et al. A cobalt-catalyzed alkene hydroboration with pinacolborane. Angew Chem Int Ed, 2014, 53: 26962700

38 Cruz TFC, Lopes PS, Pereira LCJ, et al. Hydroboration of terminal olefins with pinacolborane catalyzed by new mono(2-iminopyrrolyl) cobalt(II) complexes. Inorg Chem, 2018, 57: 8146-8159

39 Chen X, Cheng Z, Lu Z. Iron-catalyzed, Markovnikov-selective hydroboration of styrenes. Org Lett, 2017, 19: 969-971

40 Zhuang L, Ge L, Yang Y, et al. Ultrathin iron-cobalt oxide nanosheets with abundant oxygen vacancies for the oxygen evolution reaction. Adv Mater, 2017, 29: 1606793

41 Chen Y, Ji S, Sun W, et al. Discovering partially charged singleatom Pt for enhanced anti-Markovnikov alkene hydrosilylation. J Am Chem Soc, 2018, 140: 7407-7410

42 Zhu Y, Cao T, Cao C, et al. One-pot pyrolysis to N-doped graphene with high-density Pt single atomic sites as heterogeneous catalyst for alkene hydrosilylation. ACS Catal, 2018, 8: $10004-$ 10011

43 Kresse G, Furthmüller J. Efficiency of ab-initio total energy calculations for metals and semiconductors using a plane-wave basis set. Comput Mater Sci, 1996, 6: 15-50

44 Kresse G, Hafner J. Ab initio molecular-dynamics simulation of the liquid-metal-amorphous-semiconductor transition in germanium. Phys Rev B, 1994, 49: 14251-14269

45 Perdew JP, Burke K, Ernzerhof M. Generalized gradient approximation made simple. Phys Rev Lett, 1996, 77: 3865-3868 imation made simple”. Phys Rev Lett, 1998, 80: 890

47 Henkelman G, Uberuaga BP, Jónsson H. A climbing image nudged elastic band method for finding saddle points and minimum energy paths. J Chem Phys, 2000, 113: 9901-9904

48 Wang Z, Liu X, Rooney DW, et al. Elucidating the mechanism and active site of the cyclohexanol dehydrogenation on copper-based catalysts: a density functional theory study. Surf Sci, 2015, 640: 181-189

49 Cao XM, Burch R, Hardacre C, et al. An understanding of chemoselective hydrogenation on crotonaldehyde over $\operatorname{Pt}(111)$ in the free energy landscape: the microkinetics study based on firstprinciples calculations. Catal Today, 2011, 165: 71-79

50 Abild-Pedersen F, Greeley J, Studt F, et al. Scaling properties of adsorption energies for hydrogen-containing molecules on transition-metal surfaces. Phys Rev Lett, 2007, 99: 016105

51 Fernández EM, Moses PG, Toftelund A, et al. Scaling relationships for adsorption energies on transition metal oxide, sulfide, and nitride surfaces. Angew Chem Int Ed, 2008, 47: 4683-4686

52 Han M, Fu X, Cao A, et al. Toward computational design of catalysts for $\mathrm{CO}_{2}$ selective reduction via reaction phase diagram analysis. Adv Theor Simul, 2019, 2: 1800200

53 Tian X, Guo C, Zhong H, et al. Exceptional stability and chemical mechanism over spinel $\mathrm{ZnCr}_{2} \mathrm{O}_{4}$ catalyst for $\mathrm{HCl}$ oxidation to $\mathrm{Cl}_{2}$. Mol Catal, 2019, 470: 82-88

Acknowledgements This work was supported by the National Key R\&D Program of China (2018YFA0702003) and the National Natural Science Foundation of China $(21890383,21671117,21871159$ and 21901135). We thank the BL14W1 station in Shanghai Synchrotron Radiation Facility (SSRF) and 1W1B station for XAFS measurement in Beijing Synchrotron Radiation Facility (BSRF).

Author contributions $\mathrm{Xu} \mathrm{Q}$ designed and performed the experiments, analyzed the data, and wrote the paper. Guo $\mathrm{C}$ and Xiao J conducted the density functional theory calculations and contributed to the writing of the paper. Tian $S$ and Zhang $J$ assisted to carry out the experiments. Chen $\mathrm{W}$ and Zheng L helped with XAFS characterization and corresponding data analysis. Cheong WC assisted in HAADF-STEM and EDX elemental mapping characterizations. Gu L helped with the AC HAADF-STEM characterization. Liu Q and Li B helped analyze the data. Wang D and Li Y conceived the research project, analyzed the results and wrote the paper. All authors contributed to the general discussion.

Conflict of interest The authors declare that they have no conflict of interest.

Supplementary information Supporting data are available in the online version of the paper.

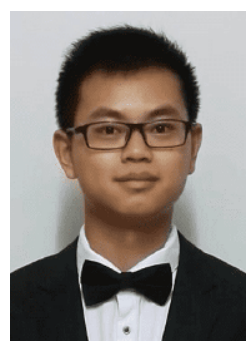

Qi Xu received his BSc degree from the University of Science and Technology of China in 2017. Now, he is a $\mathrm{PhD}$ student under the supervision of Prof. Dingsheng Wang at Tsinghua University. His research interests mainly focus on the design and fabrication of single-atomic catalysts for heterogeneous catalysis. 


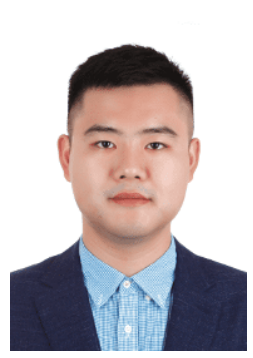

Chenxi Guo received his $\mathrm{PhD}$ degree from the Queen's University of Belfast in 2018. He is currently an Assistant Research Associate at Dalian Institute of Chemical Physics, Chinese Academy of Sciences. His research interests focus on the first principles calculations, theoretical catalysis, and rational design of catalytic materials.

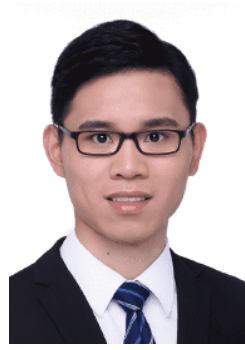

Shubo Tian received his BSc (2013), MSc (2016), and $\mathrm{PhD}$ (2019) degrees from Hebei Normal University, University of the Chinese Academy of Sciences, and Tsinghua University, respectively. $\mathrm{He}$ is currently a postdoctor in the $\mathrm{Na}$ tional University of Singapore. His research interests are focused on the syntheses and applications of isolated single-atom-site and cluster catalysts.

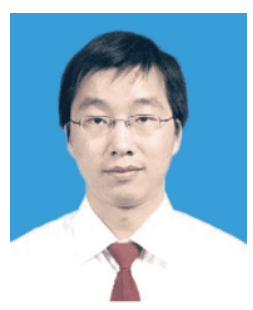

Dingsheng Wang received his BSc degree from the Department of Chemistry and Physics, University of Science and Technology of China in 2004, and his PhD degree from the Department of Chemistry, Tsinghua University in 2009, under the supervision of Prof. Yadong Li. He did his postdoctoral research at the Department of Physics, Tsinghua University, with Prof. Shoushan Fan. He joined the faculty of the Department of Chemistry, Tsinghua University in 2012.

\section{配位结构主导单原子Pt的烯烃反马氏嗍氢化催化} 性能

徐琪 $^{1 \dagger}$, 郭辰曦 ${ }^{2 \dagger}$, 田书博 ${ }^{1 \dagger}$, 张剑 ${ }^{1}$, 陈文星 $^{3}$, 张永瑧, 谷林 ${ }^{4}$, 郑黎荣 ${ }^{5}$, 肖建平 ${ }^{2}$, 刘强 ${ }^{1}$, 李必杰 ${ }^{1}$, 王定胜 ${ }^{1 *}$, 李亚拣 ${ }^{1}$

摘要 单原子催化剂的高效合理设计具有十分重要的意义, 但是目 前对于结构和性能之间的关联关系认知不足, 严重阻碍了催化剂 的发展进程. 因此, 清晰阐释金属活性中心的配位结构对于其催化 性能的影响将有利于单原子催化剂的高效设计. 本文研究报道了 单原子 $\mathrm{Pt}$ 催化剂的活性中心配位结构显著影响其在烯烃反马氏嗍 氢化反应中的性能表现. 三个 $\mathrm{O}$ 原子配位的 $\mathrm{Pt}$ 单原子催化剂 $\left(\mathrm{Pt}-\mathrm{O}_{3}\right)$ 与另外两种配位结构 $\left(\mathrm{Pt}-\mathrm{N}_{4}\right.$ 和 $\left.\mathrm{Pt}-\mathrm{O}_{2}\right)$ 相比, 表现出了更高的催化活 性, 在烯烃反马氏硼氢化反应中的转化数可达到 3288 . 密度泛函理 论计算表明, $\mathrm{Pt}-\mathrm{O}_{3}$ 催化剂具有超高活性的主要原因是其在反应相 图中具有最低的决速步能垒. 